\title{
Exciting Possibilities of Soft X-ray Emission Spectroscopy as Chemical State Analysis in EPMA and FESEM
}

\author{
H.Takahashi ${ }^{1}$, N. Handa ${ }^{1}$, T. Murano ${ }^{1}$, M. Terauchi ${ }^{2}$, M. Koike ${ }^{3}$, T. Kawachi ${ }^{3}$, T. Imazono ${ }^{3}$, \\ N.Hasegawa ${ }^{3}$, M. Koeda ${ }^{4}$, T. Nagano ${ }^{4}$, H. Sasai $^{4}$, Y. Oue ${ }^{4}$, Z. Yonezawa ${ }^{4}$ and S. Kuramoto ${ }^{4}$ \\ 1. EO Peripheral Component BU, JEOL Ltd, 1-2 Musashino, Akishima Tokyo 196-8558, Japan \\ 2. Inst. for Multidisciplinary Research for Advanced Materials, Tohoku Univ. Sendai 980-8577 Japan \\ 3. Japan Atomic Energy Agency 8-1, Umemidai, Kizu 619-0215, Japan \\ 4. Optical Components BU Device Dept., SHIMADZU Corp. 1 Nishinokyo-Kuwabaracho, Nakagyo-ku, \\ Kyoto 604-8511 Japan
}

A novel wavelength dispersive soft X-ray emission spectrometer (WD-SXES) has been developed. It covers nominally the X-ray energy range between 50 and $210 \mathrm{eV}[1,2]$. One of the characteristic features of the WD-SXES is parallel detection of the signal so that it can be used like a conventional energy dispersive spectrometer. One other feature is a high energy resolution, which is about $0.2 \mathrm{eV}$. This resolution is comparable to the one acquired with X-ray photoelectron spectroscopy or electron energy loss spectroscopy. This feature enables us to obtain various information about chemical bonding in bulk samples from observed spectra with a high energy resolution.

We have already reported a few examples obtained with the WD-SXES [3]. It is to be noted that the WD-SXES can detect Li-K emission spectrum. In the case of an anode electrode of a lithium ion battery (LIB), two types of lithium peaks are observed; one lower energy peak at $50 \mathrm{eV}$ and the other higher energy peak at $54 \mathrm{eV}$. The intensity of the former peak corresponds to the amount of charging, whereas the latter corresponds to the metallic state of lithium. The observation that the peak intensities of two different chemical states of lithium change with amount of charging suggests the usefulness of the Li-K spectrum with a high energy resolution for the characterization of lithium containing materials in LIB [4]. In the case of Kish graphite, the shape of the C-K emission spectrum is strongly orientation dependent due to the $\pi$-bonding peak with slightly higher energy than the $\sigma$-bonding peak. In the case of aluminum metal, the Al-L emission spectrum shows a shape in which the intensity gradually increases with energy and abruptly decreases to form a sharp edge at the highest energy. This shape almost perfectly corresponds to the electron density of states with the Fermi-edge. In the case of a series of rare earth fluorides, a series of spectral peaks are observed between 80 and $100 \mathrm{eV}$ as shown in Fig. 1. These are estimated to be N-band spectra influenced by outer orbital. The energy of the peaks are in the atomic order of $\mathrm{La}, \mathrm{Ce}$ and $\mathrm{Nd}$.

In this presentation, we will discuss exciting possibilities of this WD-SXES as chemical state of analysis based on our efforts performed up to this time and the results obtained so far.

\section{References:}

[1] M. Terauchi, et al., J. Electron Microscopy, 61, 1 (2012).

[2] T. Imazono, et al., Appl. Opt. 51, 2351 (2012).

[3] H. Takahashi, et al., Microscopy and Microanalysis, 19 (supple. 2), 1258 (2013).

[4] H. Takahashi, et al., Microscopy and Microanalysis, 19 (supple. 2), 1258 (2013). 


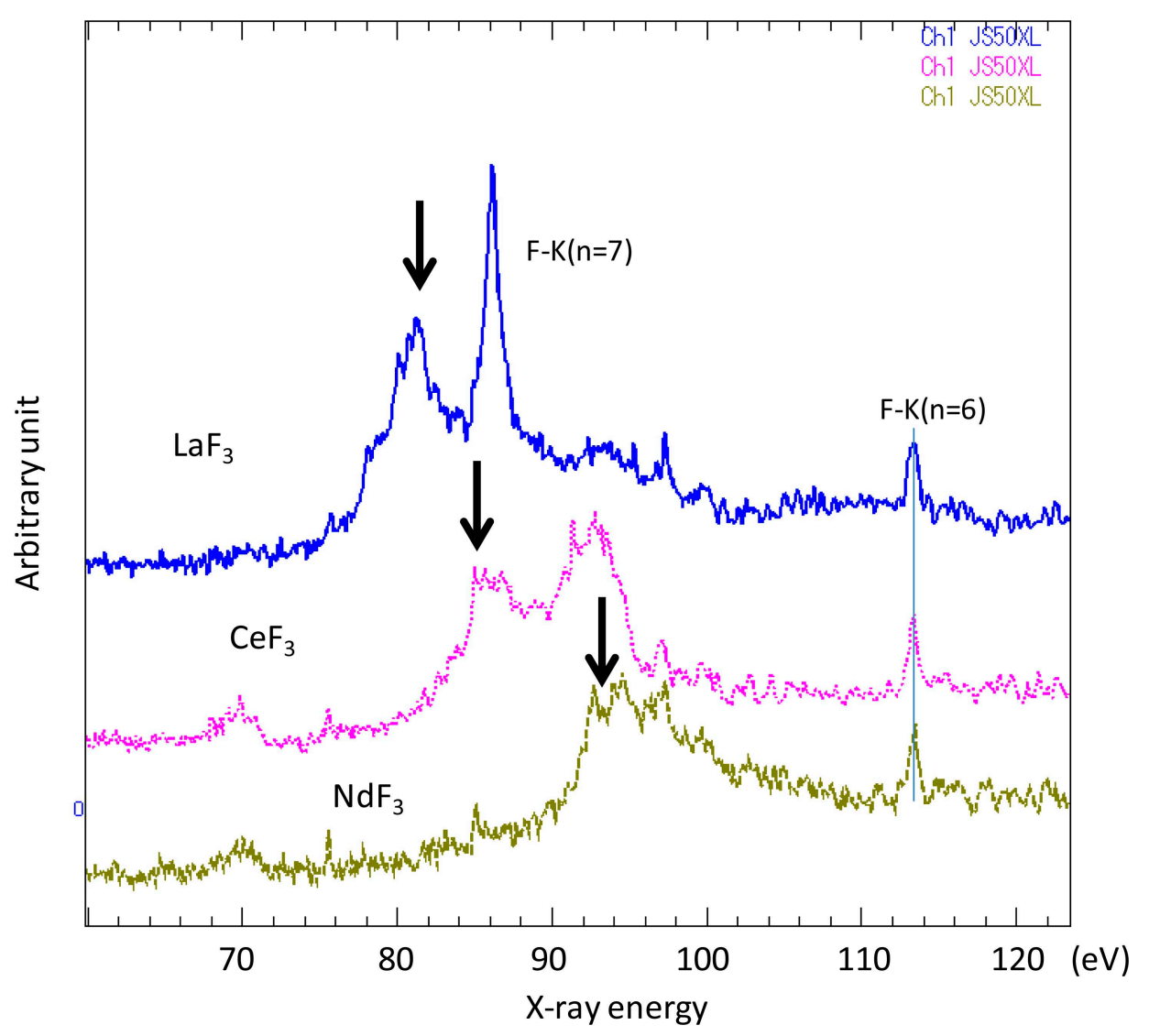

Figure 1. N-band spectra of rare earth elements $\mathrm{La}, \mathrm{Ce}$ and $\mathrm{Nd}$ 